\title{
Hebb Repetition Effects for Non-Verbal Visual Sequences: \\ Determinants of Sequence Acquisition
}

Andrew J. Johnson*, Artur Dygacz, \& Christopher Miles

Department of Psychology,

Faculty of Science \& Technology,

Bournemouth University,

Poole, UK

*Corresponding author

Address for Correspondence:

Department of Psychology

Bournemouth University

Poole House

Talbot Campus

Fern Barrow

Poole, UK

Tel: +0044 (0)1202965977

Email*: andjohnson@bournemouth.ac.uk

Word count (excluding references): 8944

Running head: Visual Hebb repetition effects

Acknowledgements: We thank Jamie Goodliffe for his technical assistance and the reviewers for their insightful comments on an earlier draft of the manuscript. 


\begin{abstract}
We report four experiments premised upon the work of Horton et al. (2008) and Page et al. (2013), and explore conditions under which the visual Hebb repetition effect is observed. Experiment 1 showed that repetition learning is evident when the items comprising the nonrepeated (filler) sequences and the repeated (Hebb) sequences are different (no-overlap). However, learning is abolished when the filler and Hebb sequences comprise the same items (full-overlap). Learning of the repeated sequence persisted when repetition spacing was increased to 6 trials (Experiment 2), consistent with that shown for verbal stimuli (Page et al., 2013). In Experiment 3 it was shown that learning for the repeated sequence is accentuated when the output motor response at test is also repeated for the Hebb sequence, but only under conditions of no-overlap. In Experiment 4, repetition spacing was re-examined with a repeated motor output response (a closer methodological analogue to Page et al., 2013). Under these conditions, the gradient of Hebb repetition learning for 6 trial repetition intervals was markedly similar to that for 3 trial intervals. These findings further support the universality of the Hebb repetition effect across memory and are discussed in terms of evidence for amodality within sequence memory.
\end{abstract}

200 words

Keywords: Hebb repetition effects; visual memory; short-term memory; serial order reconstruction 


\section{Introduction}

The Hebb repetition effect refers to the gradual acquisition of long-term memory for a sequence of items following surreptitious re-presentation of that sequence (Hebb, 1961). In a typical Hebb repetition procedure, participants undertake a series of trials requiring serialorder recall of the preceding sequence. These trials include a set of unique non-repeated sequences (known as filler sequences) and a repeated Hebb sequence, typically presented every third trial. The signature Hebb effect is demonstrated by improved recall for the Hebb sequence across repetitions. This improvement is greater than the general enhancements exhibited due to non-specific task practice effects and is, therefore, demonstrated via comparison with improvement on the filler sequences.

There has been growing interest in the Hebb repetition effect as the purported mechanism that transfers short-term sequences of phonemes held within the phonological loop into lexical representations. Indeed, Page, Cumming, Norris, McNeil, and Hitch (2013) suggest that similar processes underpin both word-form learning and the Hebb repetition effect (see also, Cumming Page, \& Norris, 2003; Mosse \& Jarrold, 2008; Szmalec, Duyck, Vandierendonck, Mata, \& Page, 2009; Szmalec, Loncke, Page, \& Duyck, 2011; Szmalec, Page, \& Duyck, 2012). Page et al. (2013) argue that learning of the Hebb sequence exhibits a number of characteristics that are similar to those responsible for novel word acquisition. First, they show that the Hebb repetition effect persists following an increase in repetition spacing. Specifically, the effect was still found with 11-intervening sequences, a manipulation analogous to the intervals between exposures to novel words. Second, participants demonstrated the ability to learn multiple Hebb sequences (an observation also reported by Hitch, Flude, \& Burgess, 2009). This is similar to how one might concurrently learn multiple novel words. Third, evidence for learning of the Hebb sequence persists over 
long retention intervals. Specifically, memory for the Hebb sequence remained evident 4months after the experiment. Moreover, data from individuals who exhibit deficits in word learning (dyslexic participants: $<10^{\text {th }}$ percentile for reading and spelling) revealed an associated generalised impairment in the Hebb repetition effect (Szmalec et al., 2011).

Whilst word learning has been strongly linked to phonological short-term memory (e.g. Baddeley, Gathercole, \& Papagno, 1998; Gathercole, 2006), therefore implicating utilisation of the phonological loop (e.g. Baddeley \& Hitch, 1974; Baddeley, 2000), the Hebb repetition effect is not confined only to verbal stimuli. Indeed, it has been shown with, for example, visual (Horton, Hay, \& Smyth, 2008; Page, Cumming, Norris, Hitch, \& McNeil, 2006), visuo-spatial (Couture \& Tremblay, 2006; Guérard, Saint-Aubin, Boucher, \& Tremblay, 2011; Tremblay \& Saint-Aubin, 2009; Turcotte, Gagnon, \& Poirier, 2005), auditory-spatial (Parmentier, Maybery, Huitson \& Jones, 2008; Lafond, Tremblay, \& Parmentier, 2010), olfactory (Johnson, Cauchi, \& Miles, 2013), and tactile (Johnson, Shaw, \& Miles, 2016) stimuli. Furthermore, the range of stimuli demonstrating the Hebb repetition effect suggest that it is underpinned by a generalised characteristic of sequence learning, and not confined to storage in the phonological loop (Page et al., 2006). This view is consistent with Hitch et al. (2009), who suggest that the episodic buffer (Baddeley, 2000) may be responsible for order memory across stimulus types (although evidence that Hebb repetition learning is not an episodic memory phenomenon is found in Gagnon, Foster, Turcotte, and Jongenelis, 2004, where densely amnesic patient SJ demonstrated Hebb repetition learning across both verbal and visuo-spatial stimuli).

The present series of experiments examine the Hebb repetition effect with visual stimuli and, therefore, the visual Hebb repetition effect reported by Horton et al. (2008) is of particular relevance. In the Horton study, participants were presented with sequences of 5faces; at test those faces were re-presented in a circular array and participants were required 
to select the items in their order of original presentation. The experiment was divided into six 3-trial epochs, where each trio of trials comprised two unrepeated filler sequences and a repeated Hebb sequence; thus the Hebb sequence was repeated every third trial. For each participant, 15 faces were used, such that the Hebb sequence and the two filler sequences each comprised a different set of 5-faces (a condition hitherto referred to as "no-stimulusoverlap'). Using this experimental procedure, Horton et al. (2008) assigned participants to one of the following stimulus conditions: upright unfamiliar faces, inverted unfamiliar-faces, and upright unfamiliar-faces under conditions of concurrent articulation (CA: aloud repetition of " $1,2,3,4$ "). Hebb repetition learning was shown for upright faces but not inverted faces; a finding they explained by psychological distinctiveness (i.e. greater efficiency in the encoding and storage of upright compared to inverted faces, e.g. see Hay, Smyth, Hitch, \& Horton, 2007). Additionally, the Hebb repetition effect was shown to be resilient to CA, a finding consistent with that found for verbal (Hitch et al., 2009), visual (Page et al., 2006), and olfactory domains (Johnson et al., 2013). Taken together, these findings argue against the view that the Hebb repetition effect is reliant upon active verbal rehearsal of the sequence stimuli (Cunningham, Healy, \& Williams, 1984, see also Oberauer \& Meyer, 2009, who argued against the need for rehearsal in demonstrating the Hebb repetition effect). In summary, Horton et al. (2008) showed that the Hebb repetition effect is found with hard-tolabel visual stimuli (i.e. faces, Ellis, 1975), even when the verbal rehearsal of any residual naming is disrupted by CA. This suggests that, as argued by Page et al. (2006) who showed the visual Hebb effect with $\mathrm{CA}$, Hebb repetition learning can occur in the absence of utilisation of the phonological loop.

The cross-modal Hebb repetition effects are of interest in suggesting the universality of the effect; however, it is also worth noting how these studies (predominantly verbal and visual stimuli) differ methodologically at the test phase. Typically, verbal learning is assessed 
via immediate serial recall (ISR); that is, ordered recall of the sequence that requires both generation of sequence items together with correct recall of their serial position (for review of ISR effects see, Bhatarah, Ward, \& Tan, 2006; Spurgeon, Ward, \& Matthews, 2014). This procedure has produced Hebb repetition learning across a range of studies (e.g. Hebb, 1961; Hitch, Fastame, \& Flude, 2005; Page et al., 2013). In contrast, non-verbal Hebb repetition learning is typically assessed via serial-order reconstruction (SOR; also termed reconstruction of order, Ward, Tan, \& Grenfell-Essam, 2010). The task requires ordered recall of the sequence following re-presentation of the sequence items at test. That is, participants recall the order without the necessity for item generation (for examples of SOR across different stimulus types see, Guérard \& Tremblay, 2008; Jones, Farrand, Stuart \& Morris, 1995; Smyth, Hay, Hitch \& Horton, 2005; Parmentier \& Jones, 2000; Ward, Avons \& Melling, 2005). As described earlier, Hebb repetition learning persists under the SOR procedure (Horton et al., 2008; see also, for example, Couture \& Tremblay, 2006; Parmentier et al., 2008). This suggests that at test, generation of the sequence items is not required to produce Hebb repetition learning, since in SOR the preceding sequence items are re-presented. Indeed there exists some debate as to whether retrieval of the sequence is required at all in order to demonstrate the Hebb repetition effect (Cohen \& Johansson, 1967a, 1967b; cf Oberauer \& Meyer, 2009). Notwithstanding this debate, Hebb repetition effects with SOR demonstrate, at least, that item generation is not a pre-requisite for learning.

Whilst the visual Hebb effect (Horton et al., 2008; Page et al., 2006) suggests universality of Hebb repetition learning, evidence to date speaks only to the general observation of improvements following sequence repetition. As noted by Hurlstone, Hitch, and Baddeley (2014) in their review of cross-modal serial-order memory studies, non-verbal studies of the Hebb repetition effect are yet to examine the full range of experimental conditions that affect learning in the verbal domain. Indeed, there remain a number of 
characteristics of the Hebb repetition effect for which the extent to which they function comparably across different modalities is unknown. One feature of interest to the current series of experiments concerns the extent of stimulus overlap between the filler and Hebb sequences. Page et al. (2013, see also Melton, 1967, who used consonants) showed that the Hebb repetition effect for sequences of 4-letter nouns was greatly reduced, and in some instances abolished, when the same nouns were employed in both the filler and Hebb sequences. Page et al. argued that using stimuli from the same set for both filler and Hebb sequences produced interference in the long-term learning of the repeated sequence. Notwithstanding the Page et al. finding, it should be emphasised that many studies report the Hebb repetition effect under conditions of full-stimulus-overlap (e.g. Couture \& Tremblay, 2006; Johnson et al., 2013, 2016; Parmentier et al., 2008). However, it remains unclear as to whether the varying effects of stimulus overlap are stimulus-specific effects or due to variations in experimental methodologies.

The present series of experiments examine the conditions under which Hebb repetition learning is found for non-verbal visual stimuli (unfamiliar faces) and begins by examining the effects of stimuli overlap. Faces are employed in the present experiment because they are non-verbal stimuli (e.g. Ellis, 1975) known to produce Hebb repetition learning (Horton et al., 2008). Indeed, our procedure follows closely that of Horton et al. (2008) who examined Hebb repetition learning following SOR for sequences of faces under conditions of no-stimulus-overlap. As described earlier, Horton et al. argued that the visual Hebb repetition effect is influenced by the within-sequence distinctiveness of the to-beremembered items (i.e. the psychological distinctiveness of the stimuli). For Experiment 1 we examine the effects of between-sequence distinctiveness on the visual Hebb repetition effect to test the extent to which the visual Hebb repetition effect is affected by stimulus overlap analogously to that found with verbal stimuli (Page et al., 2013). 


\section{Experiment 1}

Experiment 1 follows closely the design reported by Horton et al. (2008) but includes the stimulus overlap manipulation examined by Page et al. (2013, Experiment 1 and 2, and Melton, 1967). Specifically, the full-stimulus-overlap condition is tested against the nostimulus-overlap condition in a blocked repeated-measures design for which the Hebb sequence is repeated every third trial. Page et al. demonstrated that full-stimulus-overlap reduced the rate of learning for a sequence of words compared to a no-stimulus-overlap condition. We explore the extent to which the visual Hebb repetition effect is also affected by stimulus overlap between the Hebb and filler trials. Our working hypothesis is that the rate of learning of the repeated sequence will be reduced with full-stimulus-overlap compared to no-stimulus-overlap (analogous to that found with words, Page et al., 2013). This is premised on visual memory exhibiting similar serial position functions (e.g. Horton et al., 2008; Smyth et al., 2005; Ward et al., 2005; although it should be noted that Page \& Norris', 2009, model allows ordered recall without Hebb repetition learning) and error distributions (e.g. Smyth et al., 2005) to that of verbal memory. This would be in line with commonality of function for order memory across different stimulus types (see Hurlstone et al., 2014, for review).

\section{Method}

Participants. Twenty-two Bournemouth University Psychology undergraduates (mean age $=21.50$ years; 4 male and 18 female), participated in exchange for research participation credits. Ethical approval was obtained from the Bournemouth University Psychology Ethics Committee.

Materials. The unfamiliar faces were selected at random for each participant from a corpus of 60 faces (taken from Facial Recognition Technology, FERET, database (Phillips, Wechsler, Huang \& Rauss, 1998). Each face comprised 52mm x 64mm frontal images of 
Caucasian males lacking both facial hair and eye-wear. Images were greyscale and elliptically cropped to remove hair and ears. The experiment was run using the experimental software E-prime 2.0 (Psychology Software Tools, Inc.) and the face stimuli were presented on a 23 inch $(58.4 \mathrm{~cm})$ Hewlett-Packard (Palo Alto, USA) Elite Display E231 monitor.

For the full-stimulus-overlap condition, 5-upright faces were selected at random for each participant and employed in the construction of both the filler and Hebb sequences. For the no-stimulus-overlap condition, 15-upright faces were selected at random for each participant. Five faces were selected to construct the Hebb sequence, five to construct one filler sequence, and 5 to construct the other filler sequence.

Design. A 4-factor $(2 \times 2 \times 10 \times 5)$ within-participants design was employed. The first factor was experimental condition (full-stimulus-overlap and no-stimulus-overlap), the second was sequence type (filler and Hebb), the third was experimental epoch (1-10), and the fourth was serial position (1-5). The experimental condition was blocked and presented in a counterbalanced order.

Procedure. Participants were tested individually in a quiet laboratory and completed two sets of 30 trials; once with full-stimulus-overlap and once with no-stimulus-overlap. Each block began with 5-practice trials comprising a different set of 5-faces to those selected for the main experiment. Each trial was initiated by the participant via a mouse click. Trial initiation was followed by the presentation of a sequence of 5-faces, each appearing centrally on the screen for $1000 \mathrm{~ms}$ with a $1000 \mathrm{~ms}$ inter-stimulus interval (ISI). Following a $1000 \mathrm{~ms}$ retention interval (RI), the stimuli from the preceding sequence were re-presented simultaneously on the screen in a circular array. The position of each stimulus in the test array was randomised across trials. 
For the test-phase, participants were required, using the mouse, to reconstruct the presentation order of the preceding sequence by clicking on each stimulus in order. Once selected, the stimulus acquired a blue border signifying stimulus selection, and participants were unable to either change or repeat a selection. The test-phase was self-paced and successive trials did not commence until the five stimuli from the previous sequence had been selected. The task lasted approximately 20-minutes.

Analysis: Across the experiments we adopt two analytical approaches. First, we fit multilevel linear models to the data. In these models an epoch by trial type interaction may be evidence of Hebb repetition learning. A further three-way interaction may suggest that the experimental condition mediates the presence of Hebb repetition learning. Second, we fit a linear regression model to each participant in order to compute a learning gradient for both the Hebb and filler trials. We then directly compare learning gradients across conditions. Whilst, this second approach may be less sensitive than the multilevel linear model, we have included this analysis because it has been widely used in Hebb repetition learning research and enables more direct cross-study comparison.

\section{Results}

Figure 1(a-b) shows evidence for the Hebb repetition learning in the no-stimulusoverlap condition only.

- Figure 1(a-b) about here please - 
Multilevel Linear Model: We fitted multilevel linear model to the data, with proportion serial recall accuracy as the outcome variable, and experimental epoch (1-10), trial type (Hebb and filler), overlap (full stimulus overlap and no stimulus overlap), and their interactions as predictor variables. The model had random intercepts for participants and random slopes for participants by epoch, Hebb, and overlap. The results from the model are summarised in table 1.

- Table 1 about here please -

Importantly, whilst the interaction between epoch and stimulus overlap was a significant predictor $(b=.022, S E=.008, t=2.763, p=.006)$, the predicted effect of the three-way interaction between epoch, trial type, and stimulus overlap did not reach statistical significance $(b=-.021, S E=.011, t=-1.895, p=.058)$.

Gradient Analysis: The learning gradients for the filler and Hebb sequences were computed for each participant using the least squares regression method and examined via a 2-factor (2x2) within-participants ANOVA, with the factors experimental condition (fullstimulus-overlap and no-stimulus-overlap) and sequence type (filler and Hebb). The main effects of experimental condition, $F(1,21)=1.934, M S E=0.001, p=.179, \eta_{p}{ }^{2}=.084$, and sequence type, $F(1,21)=1.836, M S E=0.003, p=.190, \eta_{p}^{2}=.080$, were both non-significant. However, of theoretical interest was the significant experimental condition by sequence type interaction, $F(1,21)=6.026, M S E<0.001, p=.023, \eta_{p}{ }^{2}=.223$, demonstrating, in line with prediction, repetition learning for the no-stimulus-overlap condition only (mean gradient for the Hebb and filler conditions under no overlap $=.025$ and .006 respectively; compared to 
mean gradient for the Hebb and filler conditions under full overlap $=.003$ and .005 , respectively).

\section{Discussion}

Experiment 1 replicated the Hebb repetition effect shown with upright faces under conditions of no-stimulus-overlap (Horton et al., 2008). In addition, we have shown that this effect is abolished when the Hebb and filler sequences comprise the same faces (fullstimulus-overlap). These data suggest, therefore, that the extent to which the faces comprising the two sequence types are visually distinct is important in determining the visual Hebb effect (although it should be noted that the 3-way interaction did not reach statistical significance for the multilevel model). The results of Experiment 1 are consistent with the findings of both Page et al. (2013) and Melton (1967, who used 9-consonant visuallypresented sequences). However, our data are inconsistent with Couture and Tremblay (2006) who examined the Hebb repetition effect using visual-spatial stimuli and included a stimulus overlap manipulation. In their study sequences of dots were presented in different spatial locations followed by SOR at test. The difference in the rate of learning for the Hebb sequence between the no-stimulus overlap condition (Experiment 1: average increase in items recalled per repetition of the Hebb sequence $=0.14$ ) and a full-stimulus-overlap condition (Experiment 2: average increase in items recalled per repetition of the Hebb sequence $=$ 0.11), was minimal. This may suggest that the effects of stimulus overlap are stimulus dependent.

\section{Experiment 2}

Experiment 1 demonstrated that full stimulus overlap reduced the rate of learning for the Hebb sequence with unfamiliar faces similarly to that observed for verbal stimuli by Page et al. (2013). Historically, the Hebb repetition effect was linked to verbal repetition within the 
phonological loop (e.g. Burgess \& Hitch, 1999) and associated with lexical acquisition (Cumming et al., 2003; Moss \& Jarrold, 2008; Szmalec et al., 2009; 2011; 2012; Page et al., 2013). However, we (as suggested by others, e.g. Page et al., 2006) may now propose that this long-term sequence learning mechanism is a general feature of the memory system. To the extent that this proposed mechanism underpinning the Hebb effect is, in fact, the reflection of a universal 'learning' mechanism, then other features of the verbal Hebb repetition effect should be replicable for visual stimuli.

Page et al. (2013) showed that, for a no-stimulus-overlap condition, learning of the Hebb sequence was present despite increasing the number of filler trials between repetitions of the Hebb sequence: equivalent rates of learning were evident when the Hebb sequence was repeated every third, sixth, ninth, and twelfth sequence (although Melton, 1963, found that the effect was only observed up to repetitions every sixth trial under conditions of fullstimulus-overlap). Since our working hypothesis is that the Hebb repetition effect is present for a range of stimulus types, our second experiment tests the principle of commonality by increasing the number of filler sequences between repetitions of the Hebb sequence for visual stimuli. Thus, for Experiment 2 the rate of learning for the Hebb sequence is compared across two conditions: for one, the Hebb sequence is repeated every third trial (short-spacing) and, for the other, the Hebb sequence is repeated every sixth trial (long-spacing). Since learning was only found for the non-stimulus overlap condition in Experiment 1, the following experiment will ensure that different faces are used for the Hebb and filler trials. On the basis of Page et al.'s (2013) findings, we predict that the rate of learning will be equivalent for both Hebb repetition intervals. Such equivalence will be evidenced statistically in the multilevel linear model by (1) the 2-way sequence type by epoch interaction as a significant predictor but (2) the 3-way repetition spacing by sequence type by epoch interaction as a nonsignificant predictor. For the gradient analysis, equivalent learning for the 3-gap and 6-gap 
conditions would be evinced by a non-significant interaction for learning gradient between the sequence type condition and spacing condition. The absence of such an interaction is predicted despite evidence of an overall effect of Hebb repetition learning.

\section{Method}

Participants. Twenty-two Bournemouth University Psychology undergraduates (mean age $=21.60$ years; 5 male and 17 female), participated in exchange for research participation credits. None had participated in Experiment 1. Ethical approval was obtained from the Bournemouth University Psychology Ethics Committee.

Materials. The same set of 60 faces as described for Experiment 1 was employed. For the short-spacing condition, 15 faces were randomly sampled; from which 5 were selected for the Hebb sequence, and 5 different faces selected for each of the two filler sequences. For the long-spacing condition, 30 faces were randomly sampled; from which 5 were selected for the Hebb sequence and 5 different faces selected for each of the 5 filler sequences.

Design. A 4-factor $(2 \times 2 \times 7 \times 5)$ within-participants design was employed. The first factor was experimental condition (short- and long- spacing), the second was sequence type (filler and Hebb), the third was experimental epoch (1-7), and the fourth was serial position (1-5). For the short-spacing condition, an experimental epoch comprised one Hebb sequence and two filler sequences, and for the long-spacing condition, an experimental epoch comprised one Hebb sequence and five filler sequences. The experimental condition was blocked and presented in a counterbalanced order.

Procedure. The procedure followed closely that described for Experiment 1, with the exception that there were 21 trials in the short-spacing condition (14 filler and 7 Hebb sequences) and 42 trials in the long-spacing condition (35 filler and 7 Hebb sequences). Thus both spacing conditions comprised 7 experimental epochs. 


\section{Results}

Figure 2(a-b) shows the learning gradients for the short- and long-spacing conditions. The rate of learning for both Hebb sequences is steeper than their respective filler sequences.

- Figure 2(a-b) about here please -

Multilevel Linear Model: We fitted a multilevel linear model to the data, with proportion serial recall accuracy as the outcome variable, and experimental epoch (1-7), trial type (Hebb and filler), repetition interval (3-gap and 6-gap), and their interactions as predictor variables. The model had random intercepts for participants and random slopes for participants by epoch, Hebb, and repetition interval. The results from the model are summarised in table 2.

- Table 2 about here please -

The epoch by trial type interaction was a significant predictor $(b=-.030, S E=.012, t=-$ 2.388, $p=.017$ ), demonstrating evidence for Hebb repetition learning. However, the contribution of the three-way interaction between epoch, trial type, and repetition interval was not statistically significant $(b=.008, S E=.018, t=0.426, p=.670)$.

Gradient Analysis: The learning gradients for the filler and Hebb sequences were examined via a 2-factor $(2 \times 2)$ within-participants ANOVA, with the factors experimental 
condition (short- and long-spacing) and sequence type (filler and Hebb). The main effect of experimental condition was non-significant $F(1,21)=1.824, M S E=0.002, p=.191, \eta_{p}{ }^{2}=.080$. The main effect of sequence type was significant, $F(1,21)=12.878, M S E=0.001, p=.002, \eta_{p}^{2}$ $=.380$, (mean gradient and 95\% CI for the filler and Hebb sequences $=-.003[-.014, .007]$ and $.023[.010, .035]$, respectively) demonstrating an overall Hebb repetition learning effect. Importantly, with respect to our prediction that both spacing conditions would produce equivalent learning of the Hebb sequence, the interaction between experimental condition and sequence type was non-significant, $F(1,21)=.350, M S E=0.001, p=.561, \eta_{p}{ }^{2}=.016$.

\section{Discussion}

Experiment 2 examined the effect of repetition spacing on Hebb repetition learning for unfamiliar faces. The absence of both a sequence type by spacing interaction $(F<1)$ for the gradient analysis and 3-way interaction in the multilevel model, together with evidence for overall Hebb repetition learning, supports equivalent rates of Hebb repetition learning for the short- and long-spacing conditions. These data are consistent with Page et al. (2013) who demonstrated that the Hebb effect is evident for verbal stimuli with intervals of 5 intervening sequences. However, closer inspection of the learning gradients here for the 3-gap (.031) and 6-gap (.015) intervals suggests differences in the rates of learning (although it should be emphasised that the interaction between sequence type and spacing was non-significant and a direct comparison between the two Hebb gradients was non-significant, $t(21)=1.274, p=.217$, $r=.268$ ). This is in contrast to Page et al. (2013) who found very similar learning for 3-gap and 6-gap intervals (Experiment $1=.051$ and .066, for the 3-gap and 6-gap interval, respectively; Experiment $2=.064$ and .071 , for the 3-gap and 6-gap interval, respectively). This disparity is possibly due to methodological differences between the present study and Page et al. (2013), specifically with respect to opportunities for additional learning at the test 
phase. In Page et al. (2013), participants retrieved the sequence by writing down the words in the order of original presentation. Consequently, the motor movements required to output the Hebb sequence were also repeated and, presumably, learned. In contrast, our procedure for Experiment 2 required participants to retrieve the sequences by selecting each face in the order of original presentation from a circular array of faces. Critically, the position of each face in the test array was randomised for each trial. Thus, the motor movements required to output the Hebb sequence were different for each trial and, presumably, not learned. It is, therefore, plausible that the requirement to repeat a motor response at test for the Hebb sequence facilitated learning for Page et al. (2013).

The additive benefit of a repeated motor output for Hebb repetition learning is one potential factor underlying the observed stimulus-specific differences in Hebb repetition learning under conditions of full-stimulus-overlap. As noted earlier, there is evidence for Hebb repetition learning across a range of stimulus types, e.g., verbal (e.g. Hebb, 1961; Hitch et al., 2009; albeit reduced in Page et al., 2013, Experiment 1; Melton, 1967), visuo-spatial (Couture \& Tremblay, 2006), auditory-spatial (Parmentier et al., 2008), and tactile (Johnson et al., 2016) under conditions of full-stimulus-overlap. In contrast, Experiment 1 in the current series fails to demonstrate Hebb repetition learning for faces under those conditions. One interpretation for this disparity concerns the role of motor learning during the response phase of the task (a point noted by Cumming et al., 2003). Consider, for example, the Couture and Tremblay (2006) methodology in which the Hebb sequence comprised repeated presentation of a sequence of 7 spatial locations presented in the same order. The response for Hebb sequences required participants to reconstruct the sequence by selecting the spatial locations in the order of their presentation; thus, the order of the spatial locations was the same at both encoding and test. The motor requirement to select the spatial locations at test in the same order as that at encoding may provide additional facilitative information for learning 
of the sequence (see also Fendrich, Healy, \& Bourne, 1991). The same logic can be applied with respect to both digit (Hebb, 1961) and tactile (Johnson et al., 2016) recall, where the motor articulations required in outputting the Hebb sequence are also repeated. It is, therefore, possible that additional learning via the repeated motor response when retrieving the Hebb sequence may provide an additive benefit in learning the Hebb sequence. Consequently, we suggest that this opportunity for motor learning may explain why different Hebb repetition effects have been found cross-modally. We examine this possibility in Experiment 3; this study is the first to test directly the effects of additional motor learning at test on the Hebb effect. Additionally, we examine the extent to which, the benefit associated with motor learning on the Hebb effects can act to mitigate the negative impact of fullstimulus-overlap.

\section{Experiment 3a-b}

Experiments $3 \mathrm{a}$ and $3 \mathrm{~b}$ are designed to examine directly the impact of a repeated motor response pattern on the Hebb effect. We adopt the same experimental paradigm as that reported for Experiment 1 but manipulate the pattern of motor response required for sequence recall. This is achieved by altering the configuration of faces within the test array. For the control condition, as described for the preceding experiments, faces are positioned at random within the circular test array for each trial (change-test-configuration). The opportunity to 'learn' the motor response is thus minimised because the motor response pattern associated with the Hebb sequence (i.e. selecting the items in the order of original presentation) is different for each presentation of the Hebb sequence. For the experimental condition, the faces appear in the same location within the test array following the Hebb sequence (consistent-test-configuration). As a result, the pattern of motor response associated with recall of the Hebb sequence is repeated. Thus, the opportunity to 'learn' the motor response is 
facilitated. To the extent that repetition of the motor response pattern underpins (at least partially) the Hebb repetition effect under conditions of full-stimulus-overlap (e.g. Couture \& Tremblay, 2006; Hebb, 1961; Johnson et al., 2016; Parmentier et al., 2008), then we predict that the rate of learning for the Hebb sequence in the repeated motor response pattern condition will be steeper compared to that for the change-test-configuration (in the multilevel model, this would be demonstrated by the 3-way interaction between test configuration, sequence type, and epoch being a significant predictor). In Experiment 3a, we employ fullstimulus-overlap between the filler and Hebb sequences in order to investigate the extent to which the learning benefits of a repeated motor response compensate the diminution of learning associated with full-stimulus-overlap. Indeed, one might postulate that in studies in which learning is found under conditions of full-stimulus-overlap (e.g. Couture \& Tremblay, 2006; Hebb, 1961; Johnson et al., 2016; Parmentier et al., 2008), learning is facilitated through repetition of the same motor output at test.

In Experiment 3b, we employ no-stimulus-overlap between the Hebb and filler trials. Testing the effects of repeated motor output in conditions known to produce Hebb repetition learning (as shown in Experiments 1 and 2), allows us to examine directly the extent to which repetition of the pattern of motor response at test provides an additive learning benefit for the Hebb sequence.

\section{Method}

Participants. Experiment 3a: Twenty Bournemouth University Psychology undergraduates (mean age $=20.25$ years; 7 male and 13 female), participated in exchange for research participation credits. 
Experiment 3b: Twenty Bournemouth University Psychology undergraduates (mean age $=19.95 ; 5$ male and 15 female), participated in exchange for research participation credits.

Ethical approval was obtained from the Bournemouth University Psychology Ethics Committee. None of the participants had taken part in the preceding experiments.

Materials. For both Experiments $3 \mathrm{a}$ and $3 \mathrm{~b}$ unfamiliar faces were selected at random from the stimulus set of faces described in Experiment 1. For Experiment 3a, the same 6unfamiliar faces were selected for the Hebb and filler trials. For Experiment 3b, 18unfamiliar faces were selected at random for each session such that within each epoch a different set of 6 faces were used for the Hebb trial and each of the two filler trials.

Design. For both Experiments $3 \mathrm{a}$ and $3 \mathrm{~b}$, a 4-factor $(2 \times 2 \times 10 \times 6)$ within-participants design was employed. The first factor was experimental condition (consistent-testconfiguration and change-test-configuration), the second was sequence type (filler and Hebb), the third was experimental epoch (1-10), and the fourth was serial position (1-6). Sequence length was increased to 6-items to guard against the potential for ceiling effects when learning the Hebb sequence in the repeated motor output condition. The experimental condition was blocked and presented in a counterbalanced order.

Procedure. The procedure for both Experiments $3 \mathrm{a}$ and $3 \mathrm{~b}$ was as described for Experiment 1. Participants completed two sets of 30 trials in a counterbalanced order; once in the consistent-test-configuration condition and once in change-test-configuration condition. 


\section{Results}

Figure 3(a-d) shows the learning gradients for both experimental conditions as a function of experimental epochs. Full-stimulus-overlap is shown in Figures $3 a$ and b, whereas no-stimulus-overlap is shown in Figures $3 \mathrm{c}$ and $\mathrm{d}$.

- Figure 3(a-d) about here please -

Experiment 3a - Multilevel Linear Model: We fitted a multilevel model to the data, with proportion serial recall accuracy as the outcome variable, and experimental epoch (110), trial type (Hebb and filler), test configuration (consistent and change), and their interactions as predictor variables. The model had random intercepts for participants and random slopes for participants by epoch, Hebb, and test configuration. The results from the model are summarised in table 3.

- Table 3 about here please -

The interaction between epoch and trial type was a non-significant predictor $(b=-$ $.105, S E=.008, t=-1.347, p=.178)$ indicating no evidence of Hebb repetition learning. Nor was this effect mediated by a three-way interaction with test configuration $(b=.007, S E=$ $.011, t=0.654, p=.513)$. 
Experiment 3a - Gradient Analysis: The learning gradients for the filler and Hebb sequences were examined via a 2-factor (2x2) within-participants ANOVA, with the factors experimental condition (consistent- and change-test-configuration) and sequence type (filler and Hebb). The main effect of both experimental condition, $F(1,19)=1.51, M S E=0.001$, $p=.234, \eta_{p}{ }^{2}=.07$, and sequence type, $F<1$, were non-significant, reflecting the lack of Hebb repetition learning. The interaction between experimental condition and sequence type was non-significant, $F<1$, indicating no difference in the Hebb effect between the change- and consistent-test-configuration conditions. Despite the non-significant interaction, we assessed enhanced learning for the consistent-test-configuration condition by conducting a planned ttest between the two Hebb (consistent- and change-test-configuration) gradients. The analysis revealed a non-significant difference, $t(19)=1.17, p=.256, r=.26$ (mean gradient and $95 \% \mathrm{CI}$ for the consistent- and change-test-configuration Hebb trials $=.021[.003, .040]$ and $.008[-$ $.009, .026]$, respectively). In addition, and as expected, the two filler trials also did not differ significantly, $t<1$ (mean gradient and 95\% CI for the consistent- and change-testconfiguration filler trials $=.0107[.000, .021]$ and $.006[-.003, .015]$, respectively).

\section{Experiment 3b - Multilevel Linear Model:}

We fitted the model to the data, using the same structure as described for Experiment 3a. The results from the model are summarised in table 4.

- Table 4 about here please -

The epoch by trial type interaction was a significant predictor $(b=-.046, S E=.007, t=-$ 6.163, $p<.001)$, demonstrating evidence for Hebb repetition learning. The predicted 
contribution of the three-way interaction between epoch, trial type, and test configuration did not reach statistical significance $(b=.018, S E=.010, t=1.741, p=.082)$.

Experiment 3b - Gradient Analysis: The learning gradients for the filler and Hebb sequences were examined via the same 2-factor (2x2) within-participants ANOVA, described for Experiment 3a. The main effects of experimental condition, $F(1,19)=6.64, M S E=0.001$, $p=.019, \eta_{p}^{2}=.26$ (mean gradient and 95\% CI for the consistent- and change-testconfiguration $=.028[.020, .036]$ and $.014[.006, .021]$, respectively) and sequence type were significant, $F(1,19)=34.58, M S E=0.001, p<.001, \eta_{p}{ }^{2}=.65$ (mean gradient and $95 \% \mathrm{CI}$ for the filler and Hebb sequences $=.002[-.005, .010]$ and $.039[.029, .048]$, respectively), demonstrating overall Hebb repetition learning. Despite the striking difference in the learning gradients for the consistent- and change-test-configuration conditions (see Figure $3 \mathrm{c}$ and $3 \mathrm{~d}$ ), the interaction between experimental condition and sequence type was non-significant, $F(1,19)=3.00, M S E=0.001, p=.100, \eta_{p}^{2}=.14$ (also shown in the multilevel model, where the 3-way interaction did not reach statistical significance, $p=.082$ ). However, a planned t-test between the two Hebb learning gradients revealed that learning, in line with our prediction, was significantly steeper in the consistent-test-configuration condition, $t(19)=2.76, p=.012$, $r=.54$ (mean gradient and 95\% CI for the consistent- and change-test-configuration Hebb trials $=.051[.038, .063]$ and $.027[.014, .041]$, respectively). The two filler trials did not differ significantly, $t<1$ (mean gradient and 95\% CI for the consistent- and change-testconfiguration filler trials $=.005[-.005, .015]$ and $.000[-.011, .010]$, respectively).

Combined analysis: The data from Experiments $3 \mathrm{a}$ and $3 \mathrm{~b}$ were combined to emphasise the differing effects of stimulus overlap. The learning gradients for the filler and Hebb sequences across Experiments $3 a$ were examined via a 3 -factor $(2 \times 2 \times 2)$ mixed ANOVA. This followed the structure of the preceding ANOVA with the addition of the between-participants factor of experiment ( $3 a$ and $3 b$ ). Importantly, the ANOVA found a 
significant 2-way interaction between experiment and sequence type, $F(1,38)=10.632$, $M S E=.001, p=.002, \eta_{p}{ }^{2}=.22$. This interaction, as described above, was driven by stronger Hebb repetition effects for the no stimulus overlap used in Experiment 3b, compared to the full stimulus overlap in Experiment 3a. The 3-way interaction between experiment, experimental condition (consistent- and change-test-configuration), and sequence type (filler and Hebb) was non-significant, $F<1$.

\section{Discussion}

We proposed that repetition of the pattern of motor response at test may provide an additive benefit to learning in the Hebb repetition effect. Experiment 3a revealed no learning under the condition of full-stimulus-overlap despite a repeated pattern of motor response at test. This study, therefore, fails to explain why the Hebb effect is found, for some stimulus types, under conditions of full-stimulus-overlap (e.g. Couture \& Tremblay, 2006; Hebb, 1961; Johnson et al., 2016; Parmentier et al., 2008). In contrast, Experiment 3b, employing no-stimulus-overlap, demonstrated that learning of the Hebb sequence was accentuated when the pattern of motor response at test was repeated. Experiment $3 b$, therefore, demonstrates that stimulus overlap is a key manipulation in determining presence of the visual Hebb repetition effect. Specifically, the reduction in learning following full-stimulus-overlap cannot be reversed by invoking the same pattern of motor response at test.

The role of motor learning in the Hebb effect is considered by Cumming et al. (2003). Whilst they speculate that motor response repetition at test might facilitate learning of the sequence, they highlight the fact that learning persists when recall of the Hebb sequence requires a different motor response. This point is shown directly in the present experiment where the Hebb effect is evident for the change-test-configuration condition (albeit at a reduced level and only under conditions of no-stimulus-overlap). Indeed, Fendrich et al. 
(1991) showed that the speed with which participants typed sequences on a keypad (an implicit measure of ISR) was faster when the motor response at output was repeated. Additionally, they also demonstrated improvements in speed when the items remained the same but the motor response differed (through a modification of the keyboard configuration). Whilst our data support the motor learning reported by Fendrich et al. (1991), Experiments 3a and $3 \mathrm{~b}$ indicate that standard sequence Hebb repetition effects need to be present in order for any additive effects of motor-based Hebb repetition effects to manifest. The findings of Experiment $3 \mathrm{~b}$ thus highlight an important variable that should be controlled in future Hebb studies. Specifically, the Hebb effect can be inflated if the motor response for the Hebb sequence is also repeated. Whilst this is a methodological point, the findings of Experiment 3 have potential theoretical implications. Since learning was absent under conditions of fullstimulus-overlap (despite a repeated motor response at test) it suggests that the requirement for a repeated motor response at test cannot explain the cross-modal differences in Hebb effects. It suggests that, for example, non-verbal visual (the present experiment) and visualspatial (Couture \& Tremblay, 2006) memory systems differ with respect to the conditions in which Hebb effects are found. This is problematic for claims that a common mechanism underpins Hebb repetition learning (e.g. Hitch et al., 2009). However, such conclusions are premature given the limitations of cross-modal comparisons that are made across studies with differing methodologies.

Our demonstration of accentuated Hebb learning in Experiment $3 \mathrm{~b}$ speaks directly to the findings of Experiment 2. As described previously, the rate of learning for the Hebb sequence when repeated every six trials (6-gap) was reduced compared to the 3-gap condition (although this apparent difference was not statistically different). In contrast, Page et al. (2013, Experiments 1 and 2) reported similar learning gradients for the 3-gap and 6-gap learning conditions in the absence of stimulus overlap. However, in Page et al. (2013) there 
was potential for additional learning since participants retrieved the sequence by writing responses. Thus, the motor response at test for the Hebb sequence was repeated (by writing the same list of words in the same order). This is in contrast to our Experiment 2 where a changing-test-configuration resulted in different motor responses for the Hebb sequence. Experiment 4 is designed, therefore, to examine directly the rate of repetition learning following different repetition spacing when under conditions of repeated motor response at test.

\section{Experiment 4}

The design of Experiment 4 follows closely that described for Experiment 2 with the exception that the consistent-test-configuration is employed for the testing array. Consequently, correct retrieval of the Hebb sequence requires the same pattern of motor response at test. The recall procedure is now closely aligned to that reported by Page et al. (2013), and thus facilitates an equitable comparison between visual Hebb repetition effects in the present study and those of Page et al. (2013) for verbal stimuli. As stated for Experiment 2, equivalent learning gradients for different spacing intervals will be evidenced statistically by a non-significant interaction between the spacing condition and the sequence type condition. The absence of such an interaction is predicted together with evidence of an overall improvement in the Hebb sequence relative to filler trials.

\section{Method}

Participants. Twenty-two Bournemouth University Psychology undergraduates (mean age $=19.18$ years; 5 male and 17 female), participated in exchange for research participation credits. None had participated in the preceding experiments. Ethical approval was obtained from the Bournemouth University Psychology Ethics Committee. 
Materials. The materials were as described for Experiment 2.

Design. The design was as described for Experiment 2.

Procedure. The procedure followed closely that described in Experiment 2, with the exception that there was a consistent-test-configuration used at test for both the filler and Hebb sequences.

\section{Results}

Figure 4(a-b) shows the learning gradients for the short- and long-spacing conditions.

- Figure 4(a-b) about here please -

Multilevel Linear Model: We fitted a multilevel linear model to the data, with proportion serial recall accuracy as the outcome variable, and experimental epoch (1-7), trial type (Hebb and filler), repetition interval (3-gap and 6-gap), and their interactions as predictor variables. The model had random intercepts for participants and random slopes for participants by epoch, Hebb, and repetition interval. The results from the model are summarised in table 5.

- Table 5 about here please -

The epoch by trial type interaction was a significant predictor $(b=-.042, S E=.013, t=-$ 3.212, $p=.001$ ), demonstrating evidence for Hebb repetition learning. The three-way 
interaction between epoch, trial type, and repetition interval was not a significant predictor within the model $(b=.024, S E=.019, t=1.314, p=.190)$.

Gradient Analysis: The learning gradients for the filler and Hebb sequences were examined via a 2-factor $(2 \times 2)$ within-participants ANOVA, with the factors experimental condition (short- and long-spacing) and sequence type (filler and Hebb). The main effect of experimental condition was non-significant $F<1$. The main effect of sequence type was significant, $F(1,21)=7.26, M S E=.003, p=.014, \eta_{p}{ }^{2}=.257$, (mean gradient and $95 \%$ CI for the filler and Hebb sequences $=.003[-.008, .015]$ and .033 [.014, .053], respectively) demonstrating Hebb learning. Importantly, with respect to our prediction that both spacing conditions would produce equivalent learning of the Hebb sequence, the interaction between experimental condition and sequence type was non-significant, $F(1,21)=1.781, M S E=.002$, $p=.561, \eta_{p}{ }^{2}=.016$. In particular, we highlight the similarity of Hebb learning gradient for the 3-gap (.036) and 6-gap (.031) intervals. Moreover, as highlighted during the review process, the Hebb gradients exist in the context of quite different filler sequences (mean gradient and 95\% CI for the 3-gap and 6-gap filler sequences $=-.006[-.024, .011]$ and $.013[.001, .025]$, respectively). If one accepts that the filler gradients are not showing practice effects but instead are randomly distributed around a slope of approximately zero, one can justify a direct comparison between the 3-gap and 6-gap Hebb sequences. Consistent with the preceding analysis, such a comparison reveals no significant difference, $t(21)=0.267, p=.792$, $r=.058)$.

\section{Discussion}

Experiment 4 compared 3-gap and 6-gap repetition intervals for visual stimuli with both a no-stimulus-overlap condition and the requirement to repeat the motor response at test. As in Experiment 2, we report a main effect of sequence type (i.e. a Hebb effect) and again 
the interaction between the sequence type and repetition spacing for the gradient analysis was non-significant (as was the 3-way interaction in the multilevel model). Moreover, inspection of the learning gradients for the 3-gap (.036) and 6-gap (.031) Hebb sequences, reveal closely aligned rates of learning. These data contrast with Experiment 2 where the rate of learning for the 3-gap condition was double that of the 6-gap condition. We suggest that ensuring the same motor response at test provides a closer methodological analogue to that described by Page et al. (2013). In their study (Experiments 1 and 2) they report very similar learning gradients for 3-gap and 6-gap intervals. We have replicated that trend in Experiment 4, demonstrating further similarities in the conditions for which verbal and non-verbal-visual Hebb repetition effects are observed.

Both Experiments 2 and 4 have shown that visual Hebb repetition learning persists when the frequency of repetitions are reduced to every sixth trial. Similarly, the Hebb effect for verbal stimuli has been shown to survive increased intervals between repetitions (although the present study only examined intervals of 5-intervening sequences, whereas Page et al., 2013, examined up to 11-intervening sequences). Verbal Hebb repetition learning following longer repetition intervals has been argued to be evidence for the Hebb effect as a plausible mechanism for novel word acquisition. Our present data suggests that whilst the Hebb repetition effect may be implicated in the learning of novel words (Cumming et al., 2003; Page \& Norris, 2009; Page et al., 2013; Szmalec et al., 2009; 2011; 2012), similar mechanisms operate across modalities for non-verbal stimuli (e.g. as argued by Page et al., 2006). 


\section{General Discussion}

\section{Summary}

In the current series of experiments, we have investigated the conditions under which the visual Hebb repetition effect is found. We have shown that the rate of Hebb repetition learning is determined by the same factors as demonstrated previously for repeated verbal stimuli (as shown by Page et al., 2013). In Experiment 1, we demonstrated that the Hebb repetition effect was only found under conditions of no-stimulus-overlap and abolished when there is full-stimulus-overlap between the filler and Hebb sequences. In Experiments 2 and 4, we showed that the rate of learning for the Hebb sequence is equivalent for both 3- and 6sequence intervals. Experiment 3 showed that repetition of the motor response at test accentuates the rate of learning for the Hebb sequence, but only under conditions of nostimulus-overlap. Experiment 4 repeated the output motor response at test (providing a closer methodological match to ISR of verbal stimuli used by Page et al., 2013) and again demonstrated learning with 6-sequence repetition intervals.

Proposed accounts of the Hebb repetition effect were originally intended to explain verbal Hebb repetition learning; however, these accounts could equally be applied to visual memory. For example, Page and Norris (2009) suggest that the Hebb repetition effect is associated with the learning of a sequence 'chunk', whereas other models focus upon the cumulative matching of positional codes (Burgess \& Hitch, 2006). These mechanisms could, conceivably, operate within an amodal or modality-specific order memory system. The remainder of this section considers evidence as to whether the Hebb effect is the result of an amodal process. 


\section{Amodality in Order Memory}

Taken together, our data are broadly consistent with that body of work concerning the Hebb repetition effect for verbal stimuli (e.g. Hitch et al., 2005, 2009; Cumming et al., 2003; Cunningham et al., 1984; Hebb, 1961; Page et al., 2006, 2013). In particular, these analogous findings concern the effects of stimulus overlap between the filler and Hebb sequences, and repetition spacing (reported by Melton, 1963, 1967; Page et al., 2013). One might argue that the observation of Hebb repetition effects across modalities (e.g. Couture \& Tremblay, 2006; Johnson et al., 2013, 2016; Parmentier et al., 2008 etc.), combined with qualitatively equivalent cross-modal serial position curves (e.g. Guérard \& Tremblay, 2008; Johnson et al., 2016; Parmentier \& Jones, 2000; Smyth et al., 2005; Ward et al., 2005), analogous error distributions (Guérard \& Tremblay, 2008; Farrell \& Lewandowsky, 2004; Johnson et al., 2016; Smyth et al., 2005), and sequence recall characteristics (Cortis, Dent, Kennett, \& Ward, 2015; Spurgeon et al., 2014), are best interpreted parsimoniously via an amodal memory system. That is, rather than domain-specific memory systems, a generalised memory system supports storage for all stimulus types. By such an account, selective interference (e.g. Guérard \& Tremblay, 2008), significant evidence for modularity, could be explained through feature similarity (e.g. Brown, Neath \& Chater, 2007). Indeed, whilst selective interference effects are interpreted as strong evidence for modularity in working memory (e.g. Guérard \& Tremblay, 2008; Logie, Zucco \& Baddeley, 1990), modality-specific interference effects are abolished when the secondary task requires order memory. Specifically, in Vandierendonck (2016, see also Depoorter \& Vandierendonck, 2009) participants undertook two memory task concurrently. That is, they were presented with two lists of to-be-remembered items successively (the primary task and the secondary task), comprising either sequences of auditory-verbal or visual-spatial items. At recall, participants were tested on memory for the primary list followed by the secondary list, and were asked to either recall the order of items 
(order memory) or identify if an item had been changed in the list (item memory). Vandierendonck (2016) showed that both primary verbal and visuo-spatial memory tasks were more impaired by a secondary order memory task despite that secondary task employing stimuli from a different modality. Vandierendonck (2016) concluded that this was a demonstration of modality independent order memory, i.e., since the same mechanism is used to maintain order memory, concurrent order memory tasks are damaging to recall due to the division of resources within this amodal system.

\section{Modality in Order Memory}

In contrast, cross-modal differences (e.g. selective interference and the potential differences in the effects of stimulus overlap in the Hebb effect) can be explained by modality specific slave systems sharing functional characteristics. Indeed, Hursltone et al. (2014) state that it is "clearly parsimonious to assume that at least some core sequencing principles exist that apply across domains"; however, this does not falsify modularity "but suggest instead that the problem of serial order has been solved in similar ways across systems" (p.340). Thus, Hurlstone et al. argue that since the functioning of modular systems may have evolved over a lengthy iterative process, it is not surprising that these separate systems have evolved to operate using the most effective (and consequently analogous) methods. Using this logic, functional cross-modal similarity should not be taken as a priori evidence for amodality any more than it should be considered to support modularity.

Furthermore, data from Page et al. (2006, Experiment 2) suggest that representations of repeated visual sequences are not represented amodally, since conceptual repetitions of visual stimuli (under conditions of CA) did not produce Hebb repetition learning (however, cross-modal transfer data from letters and words in their Experiments 4 and 5 are rather more difficult to interpret). Indeed, Page et al. (2006) suggest that the most parsimonious 
explanation for their pattern of data is to suggest that parallel primacy gradients exist in the visual store, which, over repeated presentations, form sequence chunks analogous to those in the phonological loop. Similarly one could extend the principles of the latest incarnation of the Burgess and Hitch (2006) model (which employs multiple context-timing signals) from the phonological store to other systems within the working memory model.

A difficulty for a domain general/amodal interpretation of the Hebb repetition effect lies in the inconsistent findings regarding stimulus overlap between filler and Hebb sequences. Hebb repetition learning for spatial positions (Couture \& Tremblay, 2006; Parmentier et al., 2008), digits (Hebb, 1961; Hitch et al., 2009), odours (Johnson et al., 2013), and tactile stimuli (Johnson et al., 2016) is evident under conditions of full-stimulus-overlap. Whereas the present set of experiments suggests that the Hebb repetition effect is absent for non-verbal visual stimuli under conditions of full-stimulus-overlap (and also greatly reduced/abolished for verbal stimuli, Melton, 1967; Page et al., 2013). One might interpret this as evidence for modularity, with cross-modal differences in, for example, the effects of inter-trial interference on learning. However, an obvious caveat for these apparent crossmodal differences is the variations across modalities in methodology (e.g. Turcotte et al. 2005, used different sequence recall methods for verbal and visuo-spatial stimuli). Clearly, further work is needed examining spatial Hebb repetition learning. In particular, spatial Hebb effects should be compared cross-modally when employing a consistent methodology across stimulus types.

\section{Domain-Generality for Order, but not Item, Memory}

Our discussion has thus far assumed that the modality arguments encompass both order and item memory but it is possible that order and item memory are separable. Indeed, Hitch et al. (2009) suggest that whilst item information may be represented within domain- 
specific slave systems (as proposed by the Working Memory Model, e.g. Baddeley \& Hitch, 1974; Baddeley, 1986; Baddeley, 2000), order memory may be held within the episodic buffer. Such an account supports the neuropsychological data demonstrating dissociations in modality-specific memory (e.g. Darling, Della Sala, Logie \& Cantagallo, 2006; Jacquemot, Dupoux, \& Bachoud-Lévi, 2011; Vallar \& Baddeley, 1984) but also explains, through utilisation of the same store (the episodic buffer), why order memory is functionally equivalent cross-modally (see also the modality-independent disruptive effects of a secondary order memory task reported by Vandierendonck, 2016). In summary, such an account favours modular conceptualisations of memory as it allows some domain-specificity alongside some domain generality.

\section{Conclusions}

The present set of experiments has examined the visual Hebb repetition effect and reported clear similarities between the conditions under which the Hebb repetition effect is observed with visual stimuli compared to that reported for verbal stimuli (see Page et al., 2013). Our data therefore supports some commonality of function across visual and verbal Hebb repetition learning. In addition, we have shown that repeating the output pattern of motor response at test can accentuate learning and, therefore, any crossmodality/methodological comparisons should be mindful of additional routes by which the Hebb sequence can be acquired. 


\section{References}

Baddeley, A. (2000). The episodic buffer: A new component of working memory? Trends in Cognitive Sciences, 4(11), 417-423. doi:10.1016/S1364-6613(00)01538-2

Baddeley, A., Gathercole, S., \& Papagno, C. (1998). The phonological loop as a language learning device. Psychological Review, 105(1), 158-173. doi:10.1037/0033295X.105.1.158

Baddeley, A. D. (1986). Working Memory. Oxford, UK: Oxford University Press.

Baddeley, A. D., \& Hitch, G. J. (1974). Working memory. In G. A. Bower (Ed.), The Psychology of Learning and Motivation: Advances in Research and Theory (pp. 4789). New York: Academic Press.

Bhatarah, P., Ward, G., \& Tan, L. (2006). Examining the Relationship Between Free Recall and Immediate Serial Recall: The Effect of Concurrent Task Performance. Journal of Experimental Psychology. Learning, Memory \& Cognition, 32(2), 215-229. doi:10.1037/0278-7393.32.2.215

Brown, G. D. A., Neath, I., \& Chater, N. (2007). A temporal ratio model of memory. Psychological Review, 114(3), 539-576. doi:10.1037/0033-295X.114.3.539

Burgess, N., \& Hitch, G. J. (1999). Memory for serial order: A network model of the phonological loop and its timing. Psychological Review, 106(3), 551-581. doi:10.1037/0033-295X.106.3.551

Burgess, N., \& Hitch, G. J. (2006). A revised model of short-term memory and long-term learning of verbal sequences. Journal of Memory and Language, 55(4), 627-652. doi:10.1016/j.jml.2006.08.005 
Cohen, R. L., \& Johansson, B. S. (1967a). Some relevant factors in the transfer of material from short-term to long-term memory. The Quarterly Journal of Experimental Psychology, 19(4), 300-308. doi:10.1080/14640746708400106

Cohen, R. L., \& Johansson, B. S. (1967b). The activity trace in immediate memory: A reevaluation. Journal of Verbal Learning \& Verbal Behavior, 6(1), 139-143. doi:10.1016/S0022-5371(67)80064-1

Cortis, C., Dent, K., Kennett, S., \& Ward, G. (2015). First things first: Similar list length and output order effects for verbal and nonverbal stimuli. Journal of Experimental Psychology: Learning, Memory, and Cognition, 41(4), 1179-1214. doi:10.1037/xlm0000086

Couture, M., \& Tremblay, S. (2006). Exploring the characteristics of the visuospatial Hebb repetition effect. Memory and Cognition, 34(8), 1720-1729.

Cumming, N., Page, M., \& Norris, D. (2003). Testing a positional model of the Hebb effect. Memory, 11(1), 43-63.

Cunningham, T. F., Healy, A. F., \& Williams, D. M. (1984). Effects of repetition on shortterm retention of order information. Journal of Experimental Psychology: Learning, Memory, and Cognition, 10(4), 575-597. doi:10.1037/0278-7393.10.4.575

Darling, S., Della Sala, S., Logie, R. H., \& Cantagallo, A. (2006). Neuropsychological evidence for separating components of visuo-spatial working memory. Journal of Neurology, 253(2), 176-180.

Depoorter, A., \& Vandierendonck, A. (2009). Evidence for modality-independent order coding in working memory. Quarterly Journal of Experimental Psychology, 62(3), 531-549. 
Ellis, H. D. (1975). Recognizing faces. British Journal of Psychology, 66(4), 409-426.

Farrell, S., \& Lewandowsky, S. (2004). Modelling transposition latencies: Constraints for theories of serial order memory. Journal of Memory and Language, 51, 115-135. doi:10.1016/j.jml.2004.03.007

Fendrich, D. W., Healy, A. F., \& Bourne Jr, L. E. (1991). Long-Term Repetition Effects for Motoric and Perceptual Procedures. Journal of Experimental Psychology: Learning, Memory, and Cognition, 17(1), 137-151.

Gagnon, S., Foster, J. K., Turcotte, J., \& Jongenelis, S. (2004). Involvement of the hippocampus in implicit learning of supra-span sequences: The case of sj. Cognitive Neuropsychology, 21(8), 867-882. doi:10.1080/02643290342000609

Gathercole, S. E. (2006). Complexities and Constraints in Nonword Repetition and Word Learning. Applied Psycholinguistics, 27(4), 599-613.

Guérard, K., Saint-Aubin, J., Boucher, P., \& Tremblay, S. (2011). The role of awareness in anticipation and recall performance in the Hebb repetition paradigm: implications for sequence learning. Memory \& Cognition, 39(6), 1012-1022. doi:10.3758/s13421-0110084-1

Guérard, K., \& Tremblay, S. (2008). Revisiting evidence for modularity and functional equivalence across verbal and spatial domains in memory. Journal of Experimental Psychology: Learning, Memory, and Cognition, 34(3), 556-569. doi:10.1037/02787393.34.3.556

Hay, D. C., Smyth, M. M., Hitch, G. J., \& Horton, N. J. (2007). Serial position effects in short-term visual memory: A SIMPLE explanation? Memory \& Cognition, 35(1), 176-190. doi:10.3758/BF03195953 
Hebb, D. O. (1961). Distinctive features of learning in the higher animal. In J. Delafresnaye (Ed.), Brain mechanisms and learning (pp. 37-46). Oxford: Blackwell.

Hitch, G. J., Fastame, M. C., \& Flude, B. (2005). How is the serial order of a verbal sequence coded? Some comparisons between models. Memory, 13(3-4), 247-258.

Hitch, G. J., Flude, B., \& Burgess, N. (2009). Slave to the rhythm: Experimental tests of a model for verbal short-term memory and long-term sequence learning. Journal of Memory and Language, 61, 97-111. doi:10.1016/j.jml.2009.02.004

Horton, N., Hay, D. C., \& Smyth, M. M. (2008). Hebb repetition effects in visual memory: The roles of verbal rehearsal and distinctiveness. Quarterly Journal of Experimental Psychology, 61(12), 1769-1777. doi:10.1080/17470210802168674

Hurlstone, M. J., Hitch, G. J., \& Baddeley, A. D. (2014). Memory for serial order across domains: An overview of the literature and directions for future research. Psychological Bulletin, 140(2), 339-373. doi:10.1037/a0034221

Jacquemot, C., Dupoux, E., \& Bachoud-Levi, A. C. (2011). Is the word-length effect linked to subvocal rehearsal? Cortex, 47(4), 484-493.

Johnson, A. J., Cauchi, L., \& Miles, C. (2013). Hebbian learning for olfactory sequences. Quarterly Journal of Experimental Psychology, 66(6), 1082-1089. doi:10.1080/17470218.2012.729068

Johnson, A. J., Shaw, J., \& Miles, C. (2016). Tactile order memory: evidence for sequence learning phenomena found with other stimulus types. Journal of Cognitive Psychology, 28(6), 718-725. 
Jones, D., Farrand, P., Stuart, G., \& Morris, N. (1995). Functional equivalence of verbal and spatial information in serial short-term memory. Journal of Experimental Psychology: Learning, Memory, and Cognition, 21(4), 1008-1018. doi:10.1037/02787393.21.4.1008

Lafond, D., Tremblay, S., \& Parmentier, F. (2010). The ubiquitous nature of the Hebb repetition effect: Error learning mistaken for the absence of sequence learning. Journal of Experimental Psychology: Learning, Memory, and Cognition, 36(2), 515522. doi:10.1037/a001846910.1037/a0018469.supp (Supplemental)

Logie, R. H., Zucco, G. M., \& Baddeley, A. D. (1990). Interference with visual short-term memory. Acta Psychologica, 75(1), 55-74.

Melton, A. W. (1963). Implications of short-term memory for a general theory of memory. Journal of Verbal Learning and Verbal Behavior, 2(1), 1-21.

Melton, A. W. (1967). Relations between short-term memory, long-term memory and learning. In D. P. Kimble (Ed.), The Organisation of Recall: Proceedings of the Second International Interdisciplinary Conference on Learning Remembering and Forgetting (Vol. 2, pp. 24-62). New York: New York Academy of Sciences, Interdisciplinary Communications Program.

Mosse, E. K., \& Jarrold, C. (2008). Hebb learning, verbal short-term memory, and the acquisition of phonological forms in children. Quarterly Journal of Experimental Psychology, 61(4), 505-514. doi:10.1080/17470210701680779

Oberauer, K., \& Meyer, N. (2009). The contributions of encoding, retention, and recall to the Hebb effect. Memory, 17(7), 774-781. doi:10.1080/09658210903107861 
Page, M. P. A., Cumming, N., Norris, D., Hitch, G. J., \& McNeil, A. M. (2006). Repetition learning in the immediate serial recall of visual and auditory materials. Journal of Experimental Psychology: Learning, Memory, and Cognition, 32(4), 716-733. doi:10.1037/0278-7393.32.4.716

Page, M. P. A., Cumming, N., Norris, D., McNeil, A. M., \& Hitch, G. J. (2013). Repetitionspacing and item-overlap effects in the Hebb repetition task. Journal of Memory and Language, 69(4), 506-526.

Page, M. P. A., \& Norris, D. (2009). A model linking immediate serial recall, the Hebb repetition effect and the learning of phonological word forms. Philosophical Transactions of the Royal Society B: Biological Sciences, 364(1536), 3737-3753. doi:10.1098/rstb.2009.0173

Parmentier, F. B. R., \& Jones, D. M. (2000). Functional characteristics of auditory temporal—spatial short-term memory: Evidence from serial order errors. Journal of Experimental Psychology: Learning, Memory, and Cognition, 26(1), 222-238. doi:10.1037/0278-7393.26.1.222

Parmentier, F. B. R., Maybery, M. T., Huitson, M., \& Jones, D. M. (2008). The Perceptual Determinants of Repetition Learning in Auditory Space. Journal of Memory and Language, 58(4), 978-997.

Phillips, P. J., Rauss, P. J., Wechsler, H., \& Huang, J. (1998). The FERET database and evaluation procedure for face-recognition algorithms. Image and Vision Computing, 16(5), 295-306.

Smyth, M. M., Hay, D. C., Hitch, G. J., \& Horton, N. J. (2005). Serial position memory in the visual-spatial domain: Reconstructing sequences of unfamiliar faces. Quarterly 
Journal of Experimental Psychology: Section A, 58(5), 909-930. doi:10.1080/02724980443000412

Spurgeon, J., Ward, G., \& Matthews, W. J. (2014). Examining the relationship between immediate serial recall and immediate free recall: Common effects of phonological loop variables but only limited evidence for the phonological loop. Journal of Experimental Psychology: Learning, Memory, and Cognition, 40(4), 1110-1141. doi:10.1037/a0035784

Szmalec, A., Duyck, W., Vandierendonck, A., Mata, A. B., \& Page, M. P. A. (2009). The Hebb repetition effect as a laboratory analogue of novel word learning. Quarterly Journal of Experimental Psychology, 62(3), 435-443. doi:10.1080/17470210802386375

Szmalec, A., Loncke, M., Page, M. P. A., \& Duyck, W. (2011). Order or disorder? Impaired Hebb learning in dyslexia. Journal of Experimental Psychology: Learning, Memory, and Cognition, 37(5), 1270-1279. doi:10.1037/a0023820

Szmalec, A., Page, M. P. A., \& Duyck, W. (2012). The Development of Long-Term Lexical Representations through Hebb Repetition Learning. Journal of Memory and Language, 67(3), 342-354.

Tremblay, S., \& Saint-Aubin, J. (2009). Evidence of anticipatory eye movements in the spatial Hebb repetition effect: Insights for modeling sequence learning. Journal of Experimental Psychology: Learning, Memory, and Cognition, 35(5), 1256-1265. doi:10.1037/a0016566 
Turcotte, J., Gagnon, S., \& Poirier, M. (2005). The Effect of Old Age on the Learning of Supraspan Sequences. Psychology and Aging, 20(2), 251-260. doi:10.1037/08827974.20 .2 .251

Vallar, G., \& Baddeley, A. D. (1984). Fractionation of working memory: Neuropsychological evidence for a phonological short-term store. Journal of Verbal Learning and Verbal Behavior, 23(2), 151-161. doi:10.1016/S0022-5371(84)90104-X

Vandierendonck, A. (2016). Modality independence of order coding in working memory: Evidence from cross-modal order interference at recall. Quarterly Journal of Experimental Psychology, 69(1), 161-179. doi:10.1080/17470218.2015.1032987

Ward, G., Avons, S. E., \& Melling, L. (2005). Serial position curves in short-term memory: functional equivalence across modalities. Memory, 13(3-4), 308-317.

Ward, G., Tan, L., \& Grenfell-Essam, R. (2010). Examining the relationship between free recall and immediate serial recall: The effects of list length and output order. Journal of Experimental Psychology: Learning, Memory, and Cognition, 36(5), 1207-1241. doi:10.1037/a0020122 


\section{Figure Legends}

Figure 1( $a-b)$. Mean proportion correct recall scores for the filler and Hebb sequences as a function of experimental epoch (1-10) for the (a) full-stimulus-overlap and (b) no-stimulusoverlap conditions. Line of best fit depicts the learning gradient for both sequence types. Error bars denote the mean standard error.

Figure 2(a-b). Mean proportion correct recall scores for the filler and Hebb sequences as a function of experimental epoch (1-10) for the (a) short-spacing and (b) long-spacing conditions. Line of best fit depicts the learning gradient for both sequence types. Error bars denote the mean standard error.

Figure 3(a-d). Mean proportion correct recall scores for the filler and Hebb sequences as a function of experimental epoch (1-10) for the (a) full-overlap consistent-configuration, (b) full-overlap change-test-configuration, (c) no-overlap consistent-configuration, and (d) nooverlap change-test-configuration conditions. Line of best fit depicts the learning gradient for both sequence types. Error bars denote the mean standard error.

Figure 4(a-b). Mean proportion correct recall scores for the filler and Hebb sequences as a function of experimental epoch (1-10) for the (a) short-spacing and (b) long-spacing conditions. Line of best fit depicts the learning gradient for both sequence types. Error bars denote the mean standard error. 
Figure 1(a-b)
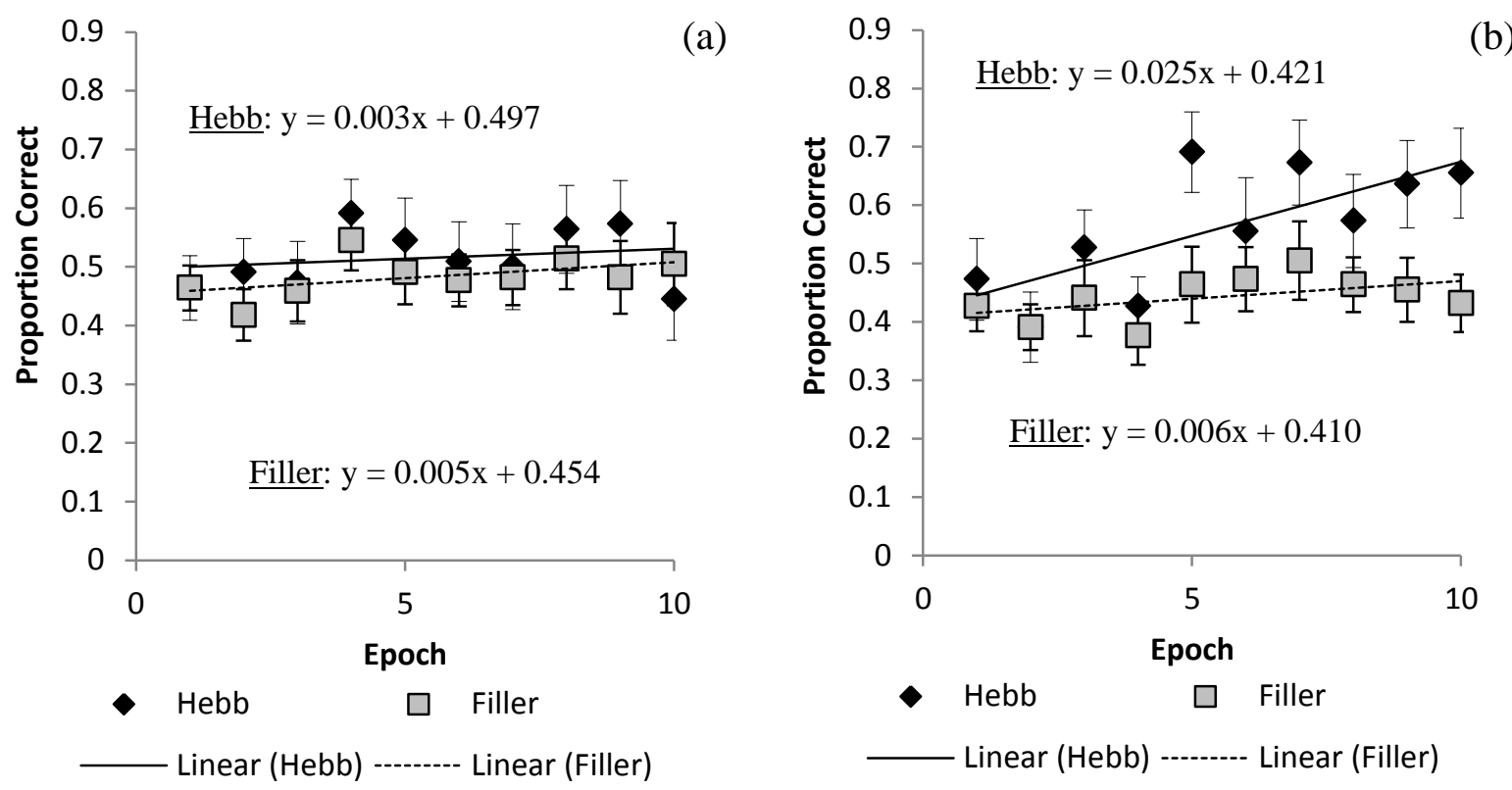
Figure 2(a-b)
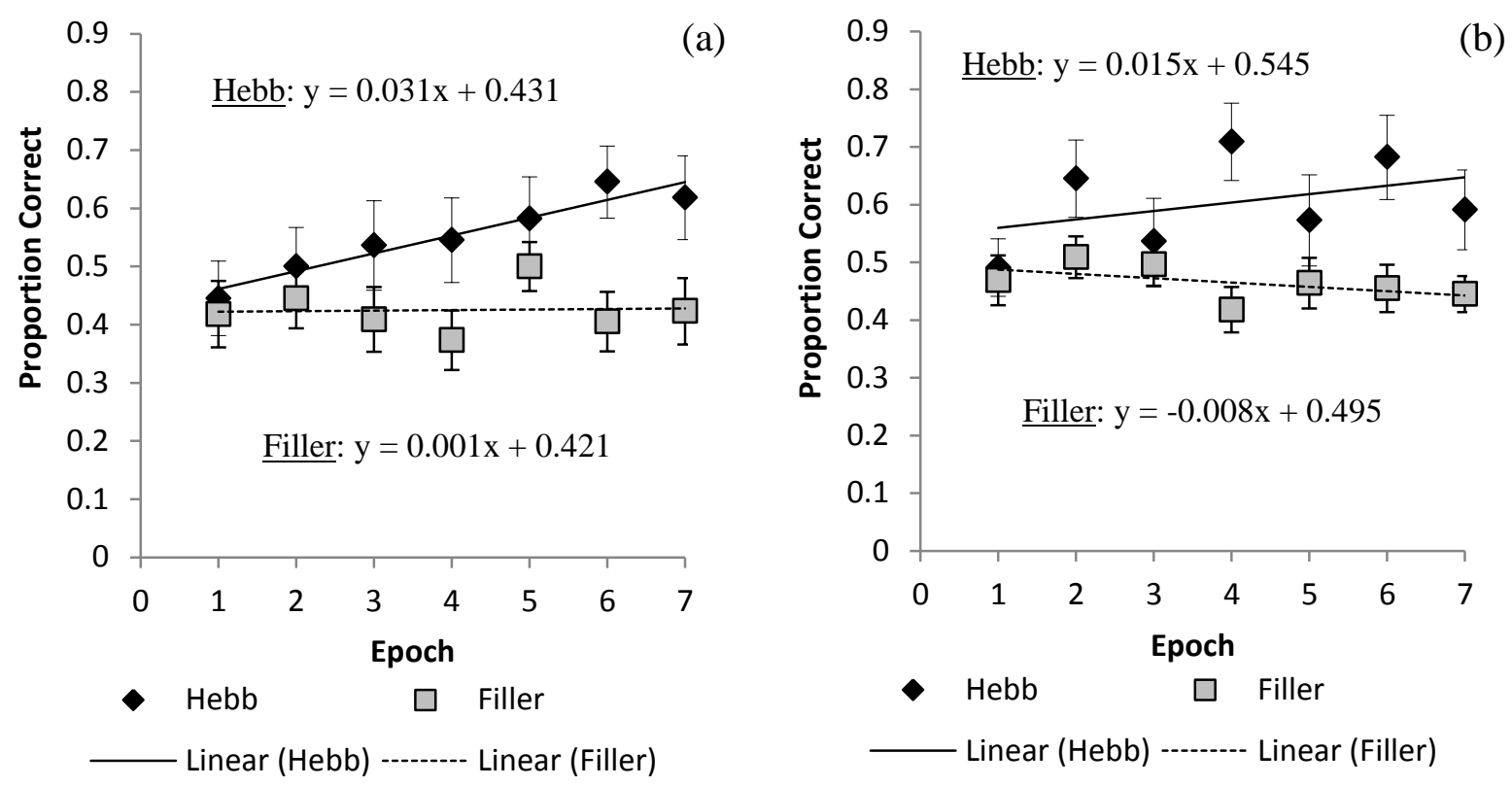
Figure 3(a-d)
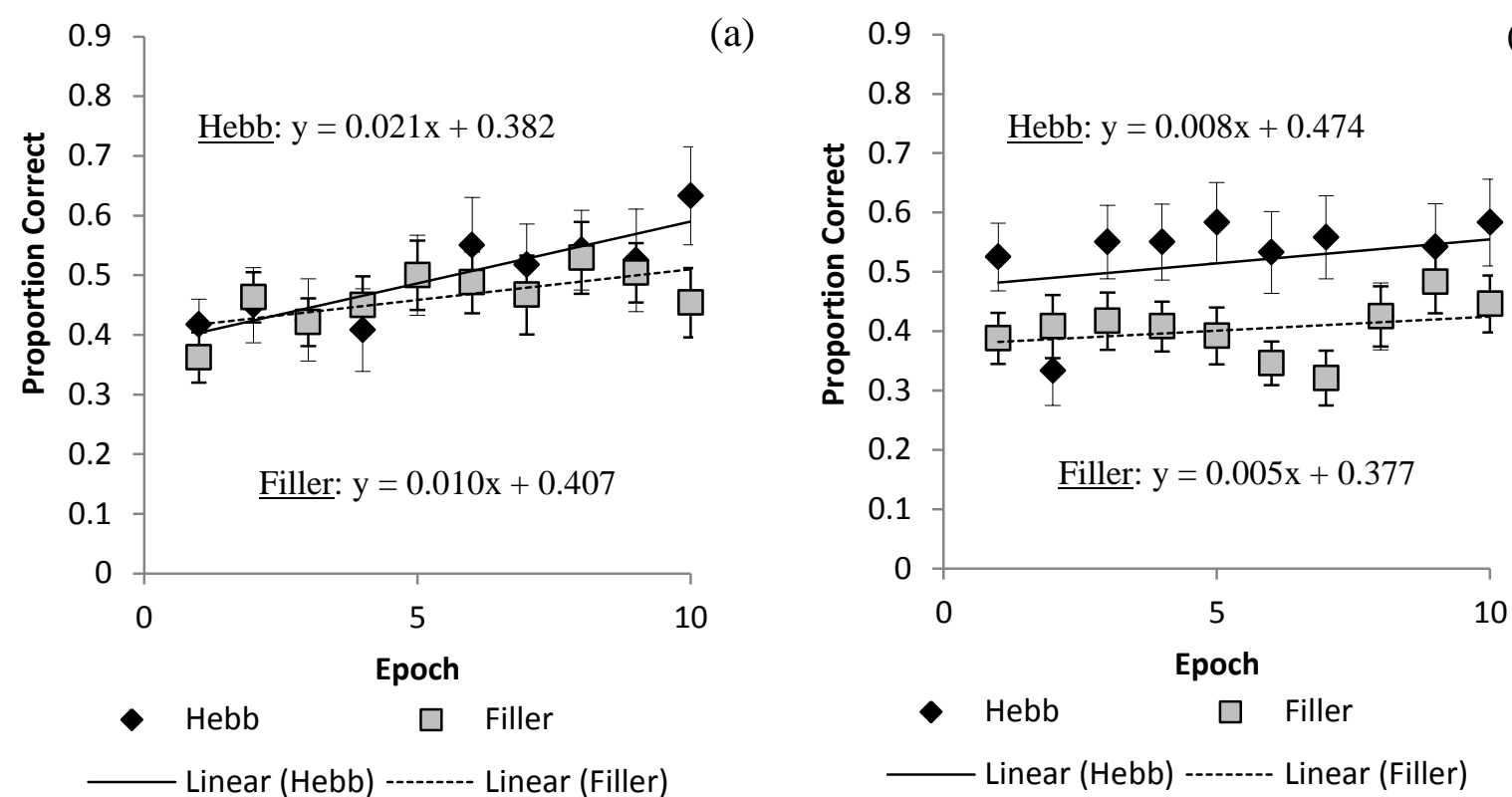

(b)

- Hebb $\square$ Filler

Linear (Hebb) -------- Linear (Filler)
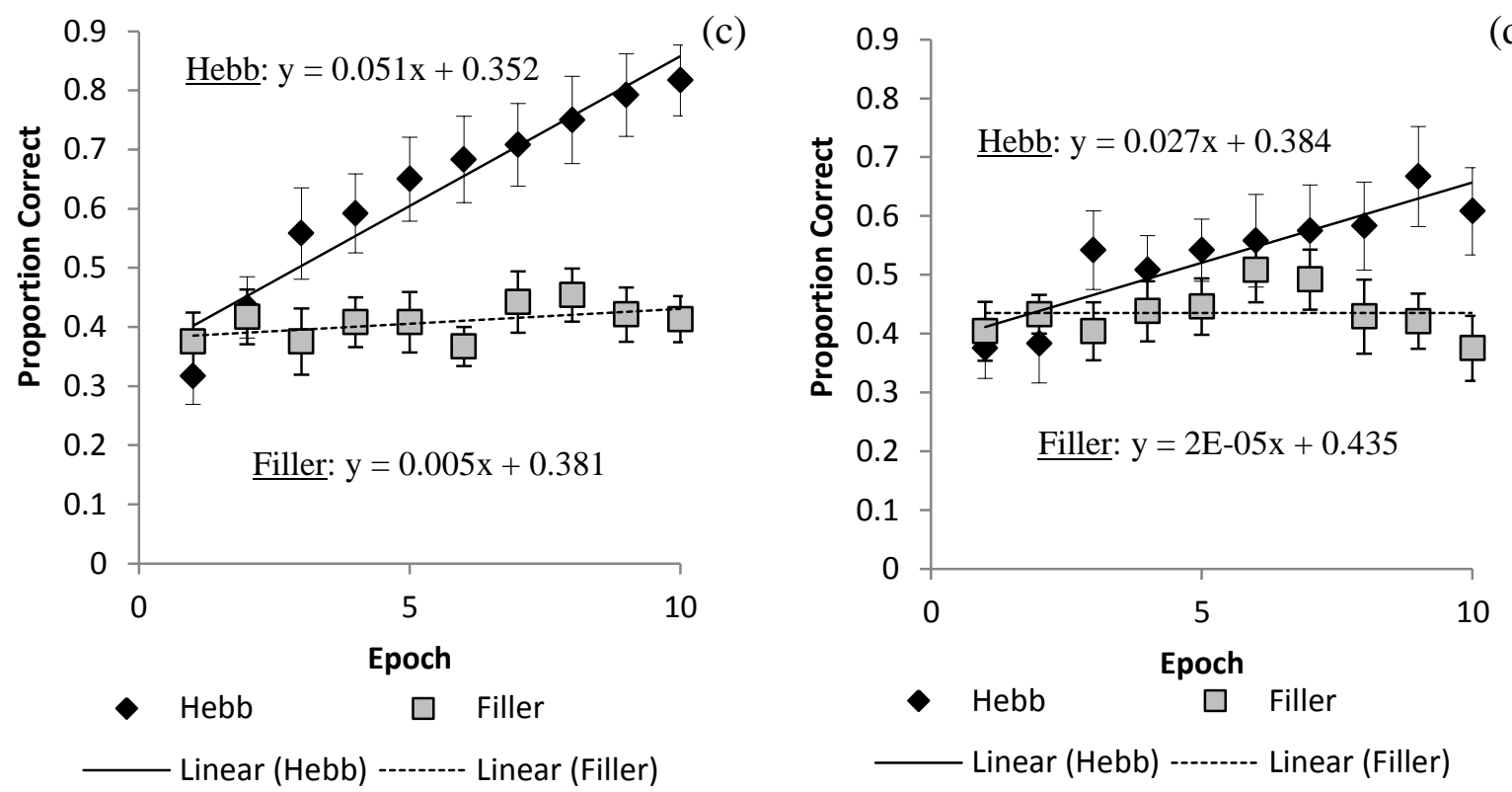


\section{Figure 4(a-b)}
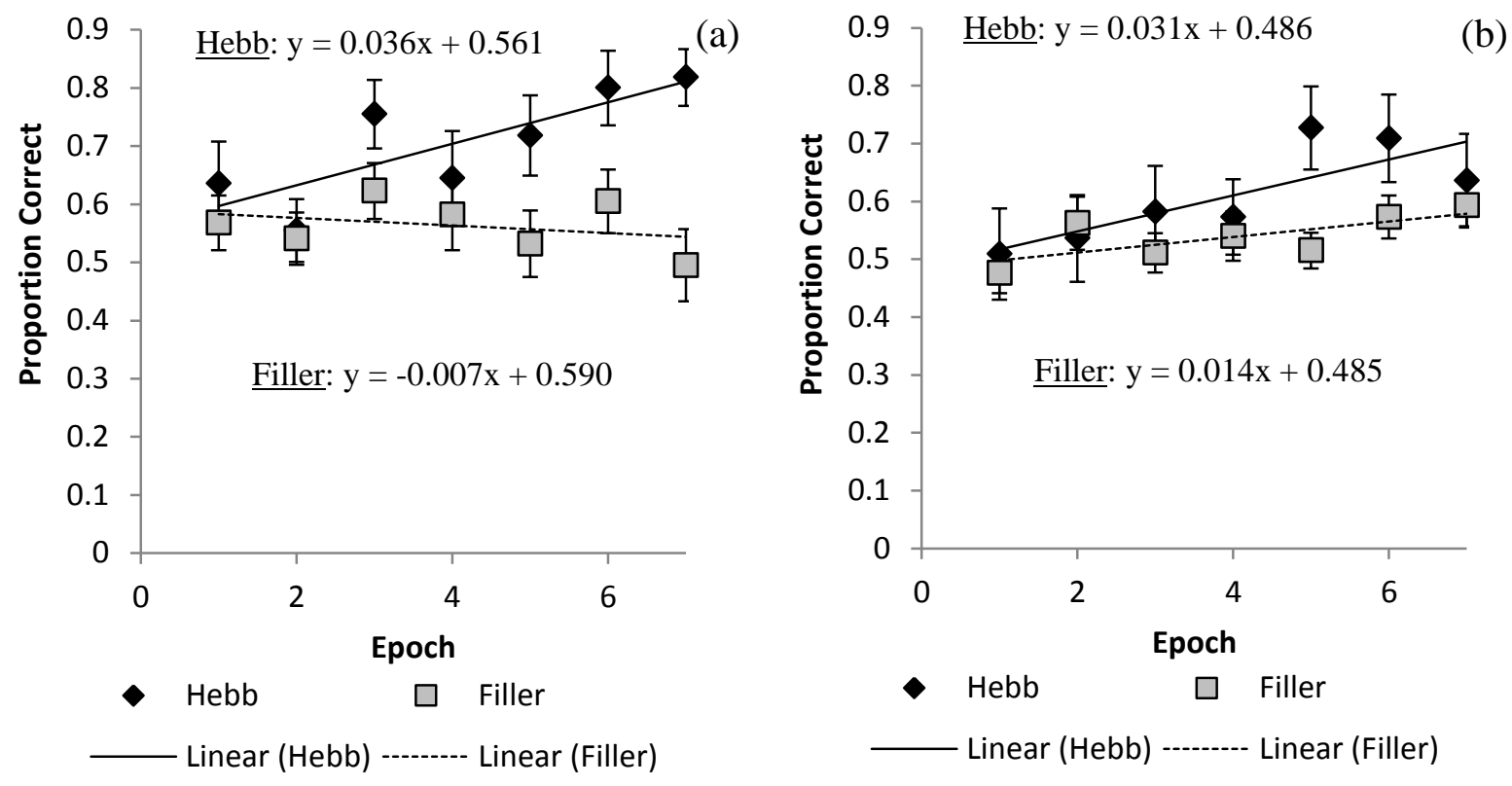
Table 1 Linear mixed model results for proportion serial position recall accuracy for epoch by trial type by overlap

\begin{tabular}{llll}
\hline Predictor & Estimate & Std. Error & $t$ value \\
\hline (Intercept) & .497 & .045 & $\mathbf{1 1 . 0 1 4}$ \\
Epoch $(1-10)$ & .003 & .006 & 0.552 \\
Trial type (Hebb vs filler) & -.043 & .052 & -0.828 \\
Stimulus overlap (full vs no) & -.076 & .054 & -1.423 \\
Epoch * Trial Type & .002 & .008 & 0.249 \\
Epoch * Stimulus Overlap & .022 & .008 & $\mathbf{2 . 7 6 3}$ \\
Trial Type * Stimulus Overlap & .032 & .070 & 0.456 \\
Epoch * Trial Type * Stimulus Overlap & -.021 & .011 & -1.895 \\
\hline
\end{tabular}

Significant $t$ values (at $p<.05$ ) are printed in bold. 
Table 2 Linear mixed model results for proportion serial position recall accuracy for epoch by trial type by repetition interval.

\begin{tabular}{llll}
\hline Predictor & Estimate & Std. Error & $t$ value \\
\hline (Intercept) & .431 & .052 & $\mathbf{8 . 2 3 0}$ \\
Epoch (1-10) & .031 & .008 & $\mathbf{3 . 4 4 9}$ \\
Trial type (Hebb vs filler) & -.010 & .060 & -0.163 \\
Repetition interval (3-gap vs 6-gap) & .114 & .064 & 1.783 \\
Epoch * Trial Type & -.030 & .012 & $\mathbf{- 2 . 3 8 8}$ \\
Epoch * Repetition interval & -.016 & .012 & -1.279 \\
Trial Type * Repetition interval & -.040 & .079 & -0.510 \\
Epoch * Trial Type * Repetition interval & .008 & .018 & 0.426 \\
\hline
\end{tabular}

Significant $t$ values (at $p<.05$ ) are printed in bold. 
Table 3 Linear mixed model results for proportion serial position recall accuracy for epoch by trial type by test configuration.

\begin{tabular}{llll}
\hline Predictor & Estimate & Std. Error & $t$ value \\
\hline (Intercept) & .383 & .044 & $\mathbf{8 . 6 3 3}$ \\
Epoch $(1-10)$ & .021 & .006 & $\mathbf{3 . 3 4 1}$ \\
Trial type (Hebb vs filler) & .024 & .051 & 0.474 \\
Test configuration (consistent vs 6-change) & .092 & .056 & 1.657 \\
Epoch * Trial Type & -.105 & .008 & -1.347 \\
Epoch * Test configuration & -.013 & .008 & -1.644 \\
Trial Type * Test configuration & -.122 & .068 & -1.792 \\
Epoch * Trial Type * Test configuration & .007 & .011 & 0.654 \\
\hline
\end{tabular}

Significant $t$ values (at $p<.05$ ) are printed in bold. 
Table 4 Linear mixed model results for proportion serial position recall accuracy for epoch by trial type by test configuration.

\begin{tabular}{llll}
\hline Predictor & Estimate & Std. Error & $t$ value \\
\hline (Intercept) & .352 & .048 & $\mathbf{7 . 3 8 7}$ \\
Epoch $(1-10)$ & .051 & .005 & $\mathbf{9 . 4 0 0}$ \\
Trial type (Hebb vs filler) & .028 & .052 & 0.539 \\
Test configuration (consistent vs 6-change) & .319 & .052 & 0.614 \\
Epoch * Trial Type & -.046 & .007 & $\mathbf{- 6 . 1 6 3}$ \\
Epoch * Test configuration & -.232 & .007 & $\mathbf{- 3 . 1 3 8}$ \\
Trial Type * Test configuration & .023 & .065 & 0.349 \\
Epoch * Trial Type * Test configuration & .018 & .010 & 1.741
\end{tabular}

Significant $t$ values (at $p<.05$ ) are printed in bold. 
Table 5 Linear mixed model results for proportion serial position recall accuracy for epoch by trial type by repetition interval.

\begin{tabular}{llll}
\hline Predictor & Estimate & Std. Error & $t$ value \\
\hline (Intercept) & .561 & .050 & $\mathbf{1 1 . 2 1 8}$ \\
Epoch (1-10) & .036 & .010 & $\mathbf{3 . 6 8 4}$ \\
Trial type (Hebb vs filler) & .029 & .062 & 0.464 \\
Repetition interval (3-gap vs 6-gap) & -.075 & .063 & -1.204 \\
Epoch * Trial Type & -.042 & .013 & $\mathbf{- 3 . 2 1 2}$ \\
Epoch * Repetition interval & -.005 & .013 & -0.346 \\
Trial Type * Repetition interval & -.029 & .083 & -.353 \\
Epoch * Trial Type * Repetition interval & .024 & .019 & 1.314 \\
\hline
\end{tabular}

Significant $t$ values (at $p<.05$ ) are printed in bold. 